\title{
The RNA-Binding Protein PCBP1 Functions as a Tumor Suppressor in Prostate Cancer by Inhibiting Mitogen Activated Protein Kinase 1
}

\author{
Yingying Zhang ${ }^{a} \quad$ Lin Meng $^{b} \quad$ Lin Xiao $^{b} \quad$ Ruiwang Liub ${ }^{b}$ Zhonghai Li ${ }^{b}$ \\ Ying-lei Wang ${ }^{\text {b }}$ \\ aDepartment of Paediatrics, Affiliated Hospital of Jining Medical University, Jining, 'Urology \\ Department, Affiliated Hospital of Jining Medical University, Jining, China
}

\section{Key Words}

Pcbp1 • Tumor suppressor • Prostate cancer • Protein kinase 1

\begin{abstract}
Background/Aims: Poly $\mathrm{r}(\mathrm{C})$ binding protein (PCBP) 1 or heterogeneous ribonucleoprotein (hnRNP) E1 is a RNA binding protein functional in multiple biological processes. In prostate cancer (PCa), PCBP1 loss was shown to be involved with increased stemness in PCacells; however,the underlying mechanism remains unclear. Method: The role of PCBP1 in prostate tumor formationwas determined by xenograft assays. Immunoprecipitationand mass spectrometry were performed to find the pathways altered after PCBP1 knockdown. Cell proliferation, migration, invasion, and soft agar colony formationassays and xenograft assays were used to determine the role of target protein pathogenesis regulation and formation of PCa. QRT-PCR was performedto quantify relative mRNA expression. Results: The expression of mitogen activated protein kinase 1 (MAPK1) or extracellular signal regulated kinase 2 (ERK2) was increased following PCBP1 loss. Attenuation of MAPK1 inhibited in vitro and in vivo tumorigenicity and metastasis in PCa cell line, PC3. Overexpression of MAPK1 in the PC3 cells increased the tumorigenicity and metastasis. Analysis of PCBP1 and MAPK1 mRNA levels in 25 PCa patients compared to tumor-adjacent normal tissue confirmed an inverse correlation between PCBP1 and MAPK1 expression. Conclusions: PCBP1 can act as a suppressor of tumor in prostate epithelial cells by inhibiting MAPK1 expression.
\end{abstract}




\section{Cellular Physiology Cell Physiol Biochem 2018:48:1747-1754 \begin{tabular}{ll|l} 
and Biochemistry Published online: August 2, 2018 & $\begin{array}{l}\text { (c) } 2018 \text { The Author(s). Published by S. Karger AG, Basel } \\
\text { www.karger.com/cpb }\end{array}$
\end{tabular} \\ Zhang et al.: RNA-Binding Protein PCBP1 Functions as a Tumor Suppressor in Prostate Cancer}

\section{Introduction}

Prostate cancer (PCa) is one of the major causes of cancer mortality. In the last few decades, PCa has exhibited steady progress (World Health Organization, 2012). Although research advances has been made, little is known about the underlying pathogenic mechanisms of the generation and maintenance of cancer stem cells which potentiate tumor recurrence and metastasis [1-4].

Recently studies has shown that the RNA binding protein, poly $\mathrm{r}(\mathrm{C})$ binding protein (PCBP)1 also calledheterogeneous nuclear ribonucleoprotein (hnRNP) E1 is central to maintenance of stemness in prostate cancer cells [5]. It was shown that there is progressive loss of PCBP1 protein expression after TGF- $\beta$ treatmentusing LNCaP and DU145 prostate cancer cells, which in turn contributes to the $\mathrm{CD} 44^{+} \mathrm{CD} 24{ }^{-} \mathrm{CD} 133^{+}$prostate stem cells enrichment and maintenance [5]. However, the precise mechanism regulating theloss of PCBP1 expression is not known.

We thus decided to adapt a prospective way of identifying changes in gene product postloss of PCBP1 expression and whether such changes contribute to prostate tumor formation and metastatic progression. Our experiments show that enforced loss of PCBP1 expression causes upregulation of mitogen-activated protein kinase 3 (MAPK3) along with other genes. In addition, our experiments show that MAPK3 is central to tumorigenesis and metastatic progression in $\mathrm{PCa}$.

\section{Materials and Methods}

\section{Cell lines, mouse handling, and reagents}

Thestudy was performed strictly according to the recommendations in the Guide for the Care and Use of Laboratory Animals of the National Institutes of Health. The protocol of animal experiments was approved by the Committee on the Ethics of Animal Experiments of Affiliated Hospital of Jining Medical University. Sodium pentobarbital was used to anesthetize the animal when all surgery was performed.To minimize suffering, all efforts were made. PC3 and CosM6 cell lines were obtained from American Type Culture Collection (ATCC;Manassas,VA, USA). PC3 and CosM6 cells were culturedin DMEM culture medium supplemented with $10 \%$ fetal bovine serum, and $10 \mathrm{ml} / \mathrm{L}$ of $100 \times$ antibiotic-antimycotic solution. All cells were maintained at $37^{\circ} \mathrm{C}$ in a humidified atmosphere which contained $5 \% \mathrm{CO}_{2}$.

Tissue samples, processing, and ethical considerations

Fresh-frozen and paraffin-embedded prostate cancer tissue specimens and corresponding adjacent normal prostate tissue samples were obtained from 25 Chinese patients at the Affiliated Hospital of Jining Medical University between 2014 and 2015. All cases were included post review by pathologist and only where complete clinical pathology and follow-up data was available. The study protocol was approved by the Institutional Review Board of the Affiliated Hospital of Jining Medical University, China. Freshly harvested samples were immersed in RNAlater (Life Technologies, Shanghai, China) before snap freezing within 30 minutes post-surgery and stored in liquid nitrogen until further use.

\section{Plasmid constructs}

For shRNA transfection, PC3 or CosM6 cells were transfected with pGIPZ-PCBP1 shRNA (OriGene, Rockville, MD, USA)or pGIPZ-GLB1, or PC3 cells with pGIPZ-MAPK1(Origene) using Lipofectamine LTX transfection reagent (Life Technologies, Shanghai, China). To obtain the stable transfection cells, puromycin was used to select the post-transfection cells. The stable transfection cells were collected and used for indicated downstream experiments. Human MPAK1 plasmid in PCMV6-AC-GFP backbone was obtained from Origene. According to the manufacturer's instructions,the Lipofectamine LTX reagent (Life Technologies, Shanghai, China) was used to transfect the PC3 cells $\left(4 \times 10^{5}\right)$ with the MAPK1 construct. Cells were diluted 1:10 after $48 \mathrm{~h}$ of transfection and subsequently selected with G418 $(250 \mu \mathrm{g} / \mathrm{ml})$ (Teknova, Hollister, CA, USA) for approximately 2 weeks.

\section{KARGER}




\section{Cellular Physiology Cell Physiol Biochem 2018;48:1747-1754 \\ \begin{tabular}{l|l|} 
and Biochemistry Published onlıne: August 2, 2018 & $\begin{array}{l}\text { C) } 2018 \text { The Author(s). Published by S. Karger AG, Basel } \\
\text { www.karger.com/cpb }\end{array}$ \\
\hline
\end{tabular}}

Zhang et al.: RNA-Binding Protein PCBP1 Functions as a Tumor Suppressor in Prostate Cancer

\section{Cell lysis and immunoprecipitation}

To perform the immunoblot analysis and immunoprecipitation, Pierce IP lysis buffer (ThermoFisher Scientific, Shanghai, China) was used to lyse the cells. The lysis buffer containedmini protease inhibitor cocktail(Roche Diagnostics, Indianapolis, IN, USA) and phosphatase inhibitor cocktails 2 and 3 (SigmaAldrich Shanghai Trading Co Ltd, Shanghai, China). And Pierce Crosslink Immunoprecipitation kit (ThermoFisher Scientific, Shanghai, China) was obtained to perform immunoprecipitation as per manufacturer's protocolusing either rabbit anti-GFP antibody (Santa Cruz Biotechnology, Shanghai, China).

\section{Mass spectrometry}

For mass spectrometry, immunoprecipitated protein containing beads were lysed using NET buffer [50 mmol/L Tris-HCl, (pH 7.4), 150 mmol/L NaCl, 0.1\% NP40, 1 mmol/L EDTA, 0.25\% gelatin, 0.02\% sodium azide, $1 \mathrm{mmol} / \mathrm{L}$ phenylmethylsulfonyl fluoride, and 1\% aprotinin]. The obtained lysates were centrifuged using $15,000 \times \mathrm{g}$ at $4^{\circ} \mathrm{C}$ for 30 minutes. PBS was used to dialysis of the collected supernatant, $5 \mathrm{mM}$ Tris 2-carboxyethyl phosphine (TCEP) was used to reduce the proteins and $10 \mathrm{mM}$ iodoacetamide was used to alkylate the proteins. Samples were digested with trypsin (Promega) in a 1:50 ratio at room temperature for 12 hours. The Ultra MicroTIP Columns (The Nest Group, Southborough, MA, USA) and theSpeedVac concentrator were used to desalt and dry the peptides. Dried peptides were resolubilized in $20 \mu \mathrm{L}$ HPLC grade water containing $0.1 \%$ formic acid. Thelinear ion trap LTQ mass spectrometer (Thermo Electron, San Jose, CA) equipped with a nanoelectrospray ion source (Thermo Electron) coupled to an Agilent 1100 micro HPLC system carried out the sample analysis. Peptides were loaded with a cooled Agilent autosampler on a $2 \mathrm{~cm}$ long pre-column filled with C18 resin (Magic C18 AQ $5 \mu \mathrm{m}$; MichromBioresources, Auburn, CA, USA). A linear gradient of 80 minutes from $5 \%$ to $40 \%$ acetonitrile in $\mathrm{H}_{2} \mathrm{O}$ with $0.1 \%$ formic acid was used to separate peptides on a $10 \mathrm{~cm}$ long fused silica emitter packed with C18 resin spraying directly into the mass spectrometer at a flow rate of $0.5 \mu \mathrm{l} /$ minute. The MS instrument was operated in positive ion mode. To acquire one MS scan followed by three collision induced dissociation MS/MS scans, the data-dependent acquisition mode was set. The MS full scans were recorded over a mass range of 400-1600 m/z. Dynamic exclusion was enabled, the repeat count was set to 2 and the exclusion duration to 30seconds. Further MS conditions were set as following: spray voltage $1.95 \mathrm{kV}$, transfer capillary temperature $230^{\circ} \mathrm{C}$, normalized collision energy $35 \%$, activation q 0.25 and activation time 30 milliseconds.

\section{Data analysis}

We used ReAdW with default settings to convert the acquired raw files to mzXML files and then the Sequest search algorithm was chosen to search against the monkey IPI database version 3.26. The Sequest search parameters contained the static modification of cysteine $+57.02 \mathrm{Da}$, at least one tryptic terminus and one missed cleavage was allowed. To estimate the false discovery rate in the datasets, the data were further processed using the Trans-Proteomic Pipeline TPP including PeptideProphet and ProteinProphet. A protein probability of 0.5 was set as a cutoff corresponding to a false discovery rate of approximately $5 \%$. The algorithm SignalPwas used to annotate the protein liat for secreted proteins. UniProt database and literature search was chosen to further manually curatefor secreted proteins. Functional annotation was assigned using the PANTHER Classification system. Relative enrichment or depletion in the shRNA-PCBP1 population was determined relative to shRNA-GLB1 cells and data was normalized using multiple housekeeping gene products. A fold-change (increase or decrease) above 5 was considered significant.

\section{Cell proliferation assay}

Mitochondrial colorimetric assay (MTT assay, Sigma-Aldrich, St. Louis, MO) was used to quantitate cell proliferation as per the manufacturer's recommendations. Results were represented by relative optical density (OD) and expressed as mean \pm standard deviation.

\section{In vitro migration assay}

PC3 cells stably expressing shRNA-MAPK1or GLB1were cultured without serum overnight, and then treated with mitomycin-C.All the cells were trypsinized and transferred into the upper chamber $\left(5 \times 10^{4}\right)$ well) of the Transwell ( $8 \mu \mathrm{m}$ pore size; BD Bioscience). The medium supplemented with 5\%FBS was served as thechemoattractant in the lower chamber. Then the $3 \%$ glutaraldehyde and crystal violet were used to 


\section{Cellular Physiology Cell Physiol Biochem 2018;48:1747-1754

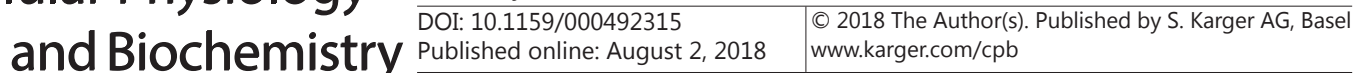 \\ Zhang et al.: RNA-Binding Protein PCBP1 Functions as a Tumor Suppressor in Prostate Cancer}

fix and stain the migratory cells respectively after. The cells stained by crystal violet were counted under an inverted microscope in 5 randomly different fields. The migration assay experiments were carried outin triplicates and repeated five individual times.

\section{In vitro invasion assay}

To determine cells invasion ability,we carried out amodified in vitro Boyden chamber invasion assay with Matrigel-coated Transwell chambers ( $8 \mu \mathrm{m}$ pore size). PC3 cells stably expressing shRNA-MAPK1or GLB1 were cultured without serum overnight, and then treated with mitomycin-C beforethe cells were trypsinized andtransferredinto the upper chamber $\left(5 \times 10^{4} /\right.$ well $)$ to rehydrated Matrigel-coated inserts (BioCoatMatrigel Invasion Chamber; Becton Dickinson) and placed in 24-well companion trans plates with DMEM and 5\% FBS (chemoattractant) to induce invasive cells to digest the coating and invade through the pores to the trans side. After 24 hours, the cells and Matrigel in the upper chambers were removed, and $3 \%$ glutaraldehyde and crystal violet were used to fix and stained the cells in the bottom trans chambers respectively. The cells stained by crystal were counted with an inverted microscope in 5 randomly different fields. All theinvasion assayexperiments were performed in triplicates and repeated three individual times.

\section{Soft agar colony formation assay}

Indicated cells $\left(1 \times 10^{3}\right)$ were re-suspended in $3 \mathrm{ml}$ of DMEM containing $0.3 \%$ agar and layered in $60 \mathrm{~mm}$ dishes containing $6 \mathrm{ml}$ of $0.5 \%$ agar beds. Cells were subsequently grown for 2 weeks before being photographed and quantified. The criterionof positive colonies was the diameter greater than $50 \mu \mathrm{m}$.

\section{Xenograft assays}

For xenograft assays, six-week-old female p53\% mice ( $\mathrm{n}=3$ per group) were sub-cutaneousinjected $10^{6}$ indicated cells at hind flank of mice. Tumor formation and metastasis of mice were assessed weekly up to 25 days using in vivo bioluminescence imaging using an IVIS Imaging System (IVIS imaging system 200, Xenogen Corporation, PerkinElmer, Waltham, USA) fitted with an ultrasensitive CCD camera.

\section{RNA extraction and quantitative real time polymerase chain reaction (qRT-PCR)}

Total RNA was tissue specimens using Trizol reagent. Real-time polymerase chain reaction (PCR) was performed using TaqMan Gene Expression probes (Life Technology, Beijing, China). GAPDH (TaqMan Assay ID: Hs02758991_g1) was used as an internal control for assessing MAPK1 and PCBP1 expression level. Data was normalized to $G A P D H$ mRNA expression and analyzed by the $-\triangle \triangle \mathrm{Ct}$ method.

\section{Statistical analysis}

Two-tailed Student's $t$ testwas used to ascertain the Statistical significance between two comparator groups. All experimentaldata were presented as mean \pm standard error of mean (SEM). The criterion of indicating statistical significance is the Pvalue $<0.05$.

\section{Results}

To understand the role of PCBP1 in prostate tumor formation, we initially did xenograft assays with PC3 cells, stably expressing either a GFP-tagged shRNA targeting beta-galactosidase (GLB1) or PCBP1. Whereas, PC3/shRNA-GLB1 cells formed small detectable tumors by Day 25 (Fig. 1A), the PC3/shRNA-PCBP1 cells formed robust tumors and metastatic lesions by day 25 (Fig. 1B). This indicated that lack of PCBP1 expression promoted both tumorigenesis and metastatic progression in prostate cancer, suggestive of $P C B P 1$ functioning as a tumor suppressor in normal prostate epithelial cells.

We next wanted to determine the pathways that are altered in the PCBP1 knockdown cells. For the same, we made stable pools of the shRNA-GLB1 and shRNA-PCBP1 cells in the CosM6 cells. Since the shRNAs were in the pGIPZ vector which also made GFP, cell lysates made from the aforementioned cells were immunoprecipitated using anti-GFP antibody (Fig. 2A). Immunoprecipitated products were processed and analyzed by mass spectrometry (Fig. 2B, C). 


\section{Cellular Physiology Cell Physiol Biochem 2018;48:1747-1754 \begin{tabular}{l|l|l} 
DOI: 10.1159/000492315 & $\begin{array}{l}\text { O } 2018 \text { The Author(s). Published by S. Karger AG, Basel } \\
\text { www.karger.com/cpb }\end{array}$ \\
\hline and Biochemistry
\end{tabular} \\ Zhang et al.: RNA-Binding Protein PCBP1 Functions as a Tumor Suppressor in Prostate Cancer}

Fig. 1. Loss of PCBP1 expression increases tumor formation and metastasis in PC3 cells. Firefly luciferase expression PC3 cells stably expressing shRNA targeting either GLB1(A) or PCBP1(B) were injected into the hind flank of $\mathrm{p} 53^{\%}$ mice. The incidence of tumor formation and metastasis was measured by luciferin injection and bioluminescence imaging.
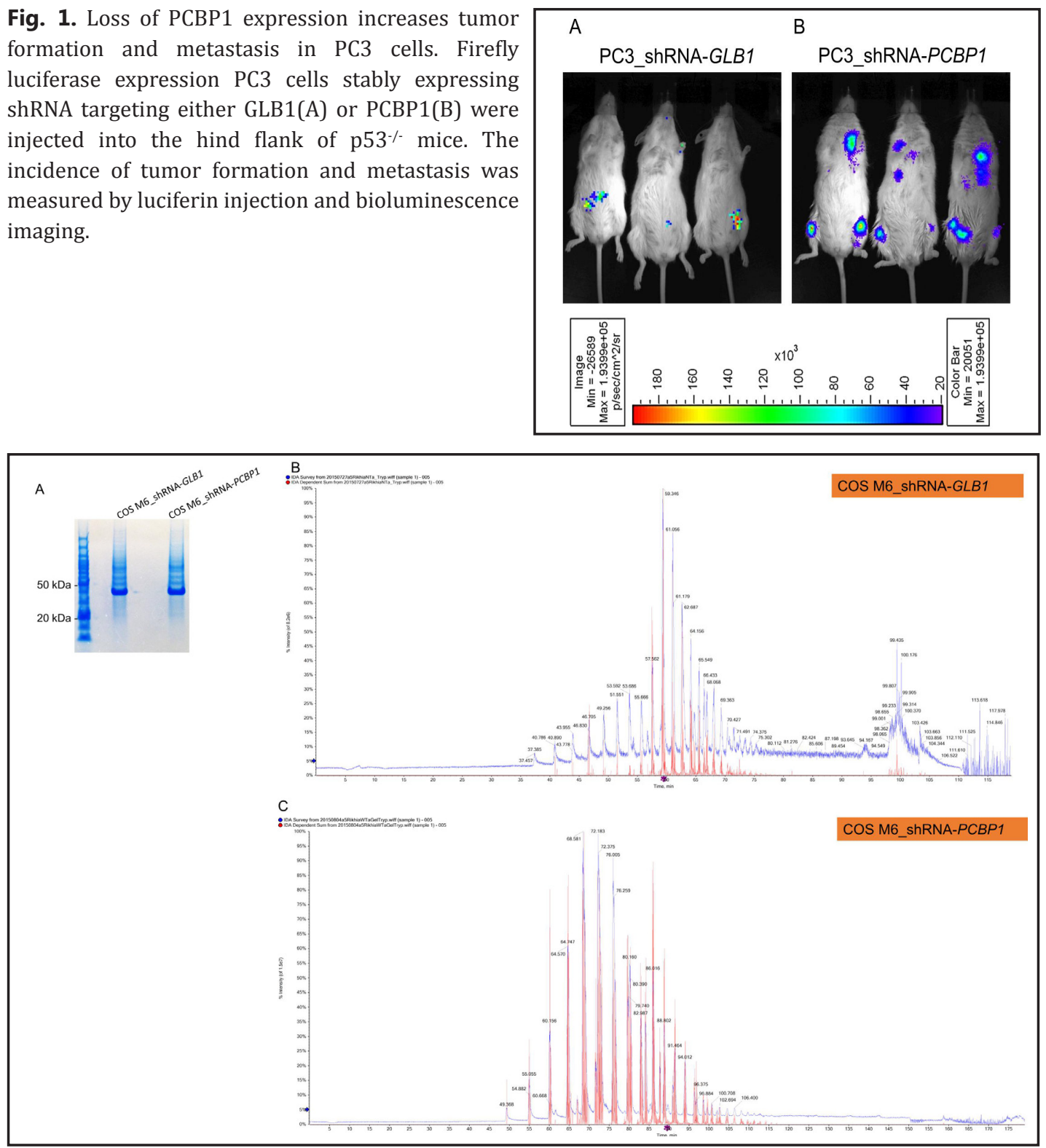

Fig. 2. Knockdown of PCBP1 potentiates tumor formation by upregulation of MAPK1 expression. (A) CosM6 cells were stably transfected with shRNA targeting GLB1 or PCBP1. Lysates prepared from stable cells were immunoprecipitated with anti-GFP antibody.Mass spectrometry was used to analyze the immunoprecipitates. (A)representsCoomassie stained gel from immunoprecipitation. (B, C) Representative electrophoretograms obtained from mass spectrometry of CosM6/shRNA-GLB1 (B) and CosM6/shRNAPCBP1 (C) cells.

Differentially expressed proteins showing at least 5 folds difference between the two cell types was analyzed (Fig. 3). Supplementary Table 1 and 2 lists the proteins enriched and peptides of those proteins identified by mass spectrometry in the PC3/shRNA-GLB1 cells. For all supplemental material see www.karger.com/doi/10.1159/000492315. Supplementary Table 3 and 4 lists the proteins enriched and peptides of those proteins identified by mass spectrometry in the PC $3 /$ shRNA-PCBP1 cells. The proteins that were most up regulated in the PC3/shRNA-PCBP1 cells were Annexin A2 isoform 5 (46.42 folds) and MAPK1, also known as p42 MAPK or ERK2 (40.76 folds). The locations of the peptides of MAPK1 within the entire MAPK1 amino acid sequence identified by the mass spectrometry are highlighted 
Fig. 3. Differential protein expression in PC3 cells harboring shRNA targeting PCBP1. Venn diagram showing proteins that were up or down regulated at least five folds and their overlap in the parental PC3 cells or the PCR3 cells harboring the shRNA/ PCBP1.

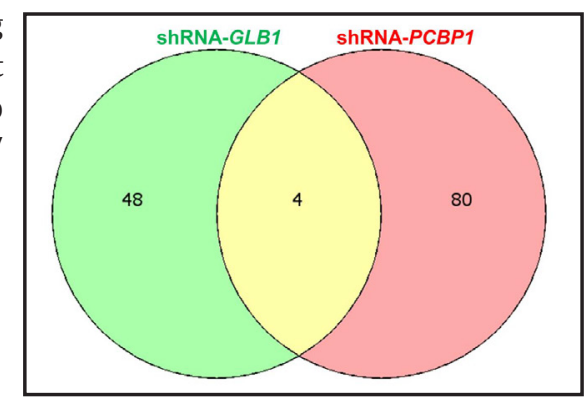

Fig. 4. Knockdown of MAPK1 impacts in vitro cell proliferation, invasion, migration, and colony formation capacity of PC3 cells. Quantification of in vitro cell proliferation (A), migration (B), invasion (C), and colony formation (D) in PC3 cells stably transfected with either GLB1 or MAPK1shRNA.

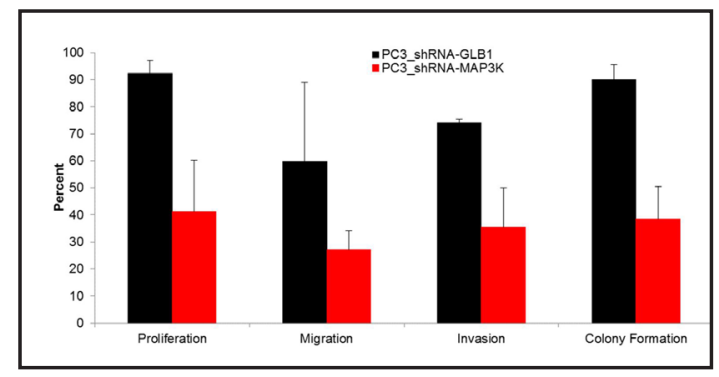

Fig. 5. MAPK1expression levels dictates tumor formation and metastasis in PC3 cells. Firefly luciferase expression PC3 cells stably expressing shRNA targeting either GLB1 (A), MAPK1(B), or overexpressing MAPK1(C) were injected into the hind flank of p531- mice. The incidence of tumor formation and metastasis was measured by luciferin injection

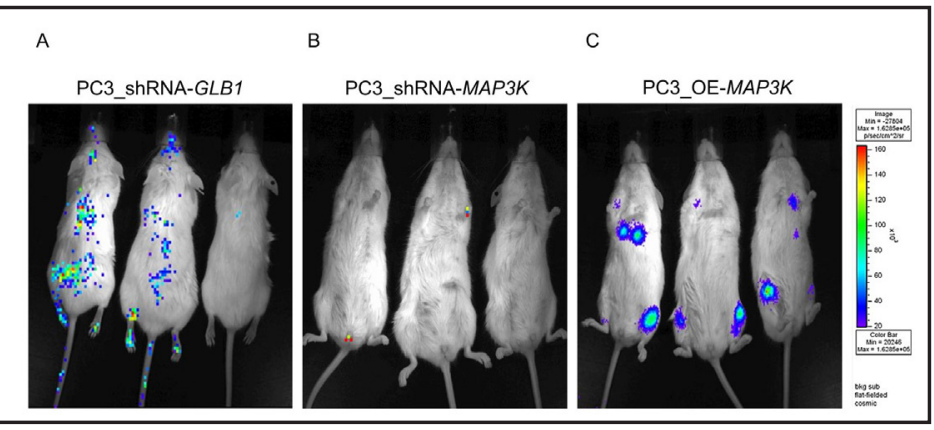
and bioluminescence imaging.

in Supplementary Table 3.

To further determine the role of MAPK1 in PCa pathogenesis regulation, we generated stable clones of PC3 expressing shRNA targeting MAPK1 and tested them in in vitro assays for proliferation, migration, invasion, and soft agar colony formation. As shown in Fig. 4, silencing of MAPK1 expression in PC3 cells significantly inhibited cell proliferation (91.3 $\pm 3.4 \%$ in PC3/shRNA-GLB1 versus $39.7 \pm 19.01 \%$ in PC3/shRNA-MAPK1, $\mathrm{p}=0.0081$ ) and colony formation $(92 \pm 3 \%$ in PC3/shRNA-GLB1 versus $37 \pm 11 \%$ in PC3/shRNA-MAPK1, $\mathrm{p}=0.0056)$, both properties associated with tumorigenic potential of cells. In addition, silencing of MAPK1 expression in PC3 cells significantly attenuated in vitro migration (59.8 $\pm 31.27 \%$ in PC3/shRNA-GLB1 versus $24.2 \pm 7.16 \%$ in PC3/shRNA-MAPK1, $\mathrm{p}=0.009$ ) and invasion $(78.5 \pm 1.4 \%$ in PC3/shRNA-GLB1 versus $38.7 \pm 12.37 \%$ in PC3/shRNA-MAPK1, p $=0.083$ ), both properties associated with metastatic dissemination of primary tumor (Fig. 4).Our results showed that MAPK1down-regulation is perhaps important to tumorigenicity and metastatic progression of these cells.

In order to understand the in vivo role of MAPK1 in formation of prostate tumor, we alsodid xenograft assays with PC3 cells, stably expressing either a GFP-tagged shRNA targeting beta-galactosidase (GLB1) or MAPK1, or overexpressing MAPK1. Whereas, PC3/ shRNA-GLB1 cells formed numerous small tumors and metastatic lesions by Day 25 (Fig. 5A), the PC3/shRNA-MAPK1 cells did not form any tumors at all (Fig. 5B). On the other hand, the PC3/MAPK1 overexpression cells formed significantly bigger tumors and metastatic lesions by day 25 (Fig. 5C). Cumulatively, this indicated that MAPK1 can potentiate both 


\section{Cellular Physiology Cell Physiol Biochem 2018;48:1747-1754

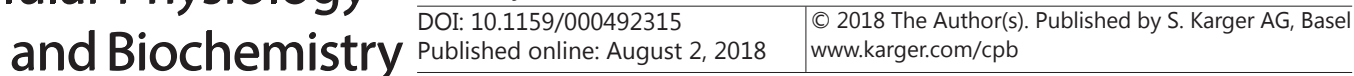 \\ Zhang et al.: RNA-Binding Protein PCBP1 Functions as a Tumor Suppressor in Prostate Cancer}

Fig. 6. PCBP1 and MAPK1 mRNA expressions are negatively correlated in prostate cancer patients. MAPK1 and PCBP1 mRNA levels were determined in tissue specimens and tumor adjacent normal tissue obtained from 25 patients. The relative expression were plotted against each other and found to be inversely correlated in these 25 patients with prostate cancer. Pearson correlation demonstrating the inverse relation between MAPK1 and PCBP1 in paired samples $(\mathrm{P}<0.05$, Pearson correlation $r=-0.9347$ ).

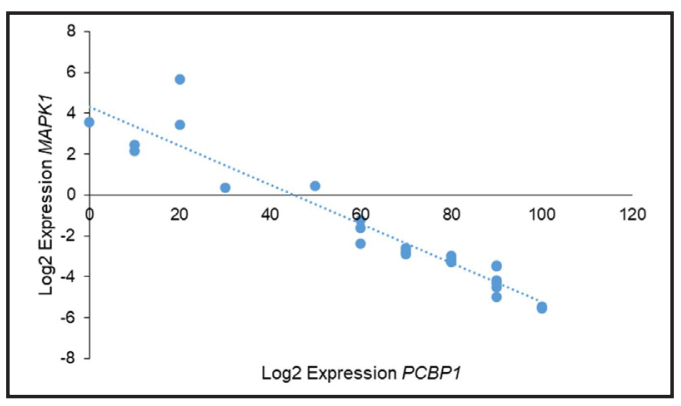

tumorigenesis and metastatic progression in prostate cancer, as revealed by lack of potent tumorigenic activity post-knockdown of MAPK1 and potent pro-tumorigenic and metastatic activity post-overexpression in the PC3 cells.

We next determined the PCBP1 and MAPK1 mRNA expressions in 25 prostate cancer patients compared to tumor adjacent normal tissue. PCBP1 and MAPK1 mRNA expressions were inversely related (Fig. 6) $(\mathrm{P}<.005$, Pearson correlation $\mathrm{r}=-0.9347)$.

\section{Discussion}

It is widely knownthat stem/progenitor cells are responsible for chemoresistance [6-8] and that lack of PCBP1 expression might be one of the central pathways underlying acquired chemoresistance [5]. Thus proto-oncogenes up-regulated as a result of repression of PCBP1 expression might be an attractive target to circumvent chemoresistance in patients with prostate cancer.

Given that $P C B P 1$ expression was being knocked down, this precluded us from doing an immunoprecipitation using anti-PCBP1 antibody. The converse strategy of overexpressing $P C B P 1$ would be counter intuitive as any proto-oncogene that is normally suppressed by PCBP1 would be further suppressed in such cases and would avoid detection. Hence, we rationalized to perform an immunoprecipitation using anti-GFP antibody. The pGIPZ shRNA construct has constitutive GFP expression and hence leveraged a tool that could be successfully used to query the mechanistic pathway in PCBP1 knockdown cells. The reason CosM6 cells was used was for robust protein synthesis and easy subculturing, providing us enough starting material for the immunoprecipitation and mass spectrometry analysis.

Our results cumulatively show that MAPK1 is up-regulated following loss of PCBP1. The MAPK pathway is well-known to be associated with tumor progression [9] and its upregulation is associated with prostate cancer too [10]. In fact, it has been shown that miR-378 which targets MAPK1 is down-regulated in PCa cells [11]. In addition it has been shown that the Her2/Raf-1/MAPK/AP-1 axis promotes castrate resistant prostate cancer [10]. Both p38 MAPK and MAPK1 has been shown to regulate E-cadherin-mediated phenotypic switching in prostate cancer [12]. ERK2 was shown as an independent prognostic marker for prostate cancer [13]. In fact, ERK2 along with the Y-box binding protein was shown to promote prostate cancer progression [13]. Our findings thus added another layer of regulation for MAPK1 by the RNA-binding protein PCBP1.

In pancreatic, ovarian and breast cancer cell line,PCBP1 has been shown to regulate the stability of the pro-oncogenic p63 transcript [14]. In addition, repression of PCBP1 it has been shown thatthe repression of PCBP1 can up-regulate translation of genes and long non-coding RNA which is required for epithelial to mesenchymal transition and metastasis in somecancers, including breast, lung, and gastric cancer [15-19]. In addition, inactivating mutations in PCBP1 has been identified in Burkitt lymphoma [20]. Thus it might be possible that down-regulation of PCBP1 represents a central point, inhibition of which is a common mechanism to increase stemness and mesenchymal cell formation in a context dependent fashion, as observed by us in the current study and the others [14-20]. 


\section{Cellular Physiology Cell Physiol Biochem 2018;48:1747-1754 \begin{tabular}{ll|l} 
and Biochemistry Published online: August 2, 2018 & $\begin{array}{l}\text { (c) } 2018 \text { The Author(s). Published by S. Karger AG, Basel } \\
\text { www.karger.com/cpb }\end{array}$
\end{tabular} \\ Zhang et al:: RNA-Binding Protein PCBP1 Functions as a Tumor Suppressor in Prostate Cancer}

\section{Disclosure Statement}

The authors declare that there is no conflict of interests regarding the publication of this paper.

\section{References}

1 Clarke MF, Dick JE, Dirks PB, Eaves CJ, Jamieson CHM, Jones DL, Visvader J, Weissman IL, Wahl GM: Cancer Stem Cells-Perspectives on Current Status and Future Directions: AACR Workshop on Cancer Stem Cells. Cancer Res 2006;66:9339-9344.

-2 Tang DG, Patrawala L, Calhoun T, Bhatia B, Choy G, Schneider-Broussard R, Jeter C: Prostate cancer stem/ progenitor cells: identification, characterization, and implications. Mol Carcinog 2007;46:1.

- Visvader JE, Lindeman GJ: Cancer stem cells in solid tumours: accumulating evidence and unresolved questions. Nat Rev Cancer 2008;8:755.

4 Rosen JM, Jordan CT: The increasing complexity of the cancer stem cell paradigm. Science 2009;324:16701673.

5 Chen Q, Cai Z, Chen Y, Gu M, Zheng D, Zhou J, Wang Z: Poly r(C) Binding Protein-1 is Central to Maintenance of Cancer Stem Cells in Prostate Cancer Cells. Cell Physiol Biochem 2015;35:1052-1061.

6 Reya T, Morrison SJ, Clarke MF, Weissman IL: Stem cells, cancer, and cancer stem cells. Nature 2001;414:105.

7 Behbod F, Rosen JM: Will cancer stem cells provide new therapeutic targets? Carcinogenesis 2005;26:703.

8 Dean M, Fojo T, Bates S: Tumour stem cells and drug resistance. Nat Rev Cancer 2005;5:275.

$\rightarrow 9$ Dhillon AS, Hagan S, Rath 0, Kolch W: MAP kinase signalling pathways in cancer. Oncogene 2007;26:32793290.

-10 Mukherjee R, Mcguinness DH, Mccall P, Underwood MA, Seywright M, Orange C, Edwards J: Upregulation of MAPK pathway is associated with survival in castrate-resistant prostate cancer. $\mathrm{Br} \mathrm{J}$ Cancer 2011;104:1920-1928.

11 Chen QG, Zhou W, Han T, Du SQ, Li ZH, Zhang Z, Shan GY, Kong CZ: MiR-378 suppresses prostate cancer cell growth through downregulation of MAPK1 in vitro and in vivo. Tumour Biol 2016;37:2095-2103.

$>12$ Ma B, Wells A: The mitogen-activated protein (MAP) kinases p38 and extracellular signal-regulated kinase (ERK) are involved in hepatocyte-mediated phenotypic switching in prostate cancer cells. J Biol Chem 2014;289:11153.

13 Imada K, Shiota M, Kohashi K, Kuroiwa K, Song Y, Sugimoto M, Naito S, Oda Y: Mutual regulation between Raf/MEK/ERK signaling and Y-box-binding protein-1 promotes prostate cancer progression. Eur Urol 2013;19:4638-4650.

14 Cho SJ, Jung YS, Chen X: Poly (C)-binding protein 1 regulates p63 expression through mRNA stability. Plos One 2013;8:e71724.

15 Wang H, Vardy LA, Tan CP, Loo JM, Guo K, Li J, Lim SG, Zhou J, Chng WJ, Ng SB: PCBP1 suppresses the translation of metastasis-associated PRL-3 phosphatase. Cancer Cell 2010;18:52-62.

-16 Chaudhury A, Hussey GS, Ray PS, Jin G, Fox PL, Howe PH: TGF-beta-mediated phosphorylation of hnRNP E1 induces EMT via transcript-selective translational induction of Dab2 and ILEI. Nat Cell Biol 2010;12:286293.

17 Hussey GS, Chaudhury A, Dawson AE, Lindner DJ, Knudsen CR, Wilce MC, Merrick WC, Howe PH: Identification of an mRNP complex regulating tumorigenesis at the translational elongation step. Mol Cell 2011;41:419-431.

18 Liu Y, Gai L, Liu J, Cui Y, Zhang Y, Feng J: Expression of poly(C)-binding protein 1 (PCBP1) in NSCLC as a negative regulator of EMT and its clinical value. Int J Clin Exp Pathol 2015;8:7165-7172.

19 Zhang ZZ, Shen ZY, Shen YY, Zhao EH, Wang M, Wang CJ, Cao H, Xu J: HOTAIR Long Noncoding RNA Promotes Gastric Cancer Metastasis through Suppression of Poly r(C)-Binding Protein (PCBP) 1 Mol Cancer Ther 2015;14:1162.

-20 Wagener R, Aukema SM, Schlesner M, Haake A, Burkhardt B, Claviez A, Drexler HG, Hummel M, Kreuz $\mathrm{M}$, Loeffler M: The PCBP1 gene encoding poly(rC) binding protein I is recurrently mutated in Burkitt lymphoma. Genes Chromosomes Cancer 2015;54:555-564. 